\title{
THE FUNDAMENTAL SOLUTIONS OF MODERATELY THICK LAMINATED ANISOTROPIC SHALLOW SHELLS
}

\author{
JIANGUO WANG $\dagger$ and KARL SCHWEIZERHOF \\ Institut für Mechanik, Universität Karlsruhe, D-76128, Karlsruhe, Germany
}

\begin{abstract}
In this paper, the partial differential equations governing moderately thick laminated anisotropic shallow shells are transferred into a set of ordinary differential equations by using the method of plane wave decomposition. With the aid of the Hörmander operator method, these ordinary differential equations are reduced to a tenth order ordinary differential equation. The fundamental solutions of moderately thick laminated anisotropic shallow shells are presented in a definite integral form. The numerical computation of the fundamental solutions is discussed in detail. Some computational formulations have been given.
\end{abstract}

\section{INTRODUCTION}

As is well known, fundamental solutions play an important role in boundary element methods which are widely used in analysis of plates, shells and some structures. The great progress has been made in the boundary element methods of isotropic body. However, to authors' knowledge, there are the few research results of boundary element methods in anisotropic plates and shells. In general, it is difficult to obtain the fundamental solutions of anisotropic body in closed form. Thus man has to use numerical fundamental solutions in boundary element methods. Lukasiewicz [1] has presented an approximate fundamental solution of orthotropic thin plates by Fourier transform technique. Wang and Huang [2] and Wang [3] have presented the fundamental solutions of orthotropic thick plates and orthotropic thin shells in a definite integral form by the Hörmander operator method [4] and the method of plane wave decomposition [5]. Wang and Huang $[2,6]$ have analyzed moderately thick orthotropic plates with boundary element methods.

With the increasing use of fibre-reinforced composite material, the structures of laminated plates and shells are widely used in engineering. Recent research shows that transverse shear deformation effects are more pronounced in anisotropic plates and shells than in isotropic plates and shells. In this paper, the fundamental solutions of laminated anisotropic shallow shells including shear transverse deformation have been presented by the use of the Hörmander method [4] and the method of plane wave decomposition [5]. The computation of the fundamental solutions is discussed in detail.

\section{BASIC EQUATIONS}

In this section, the basic equations of moderately thick laminated anisotropic shallow shells are reviewed.

The relations between the generalized displacements and the strains are

$$
\begin{array}{ll}
\epsilon_{1}=\frac{\partial u}{\partial x}+w k_{1} & \epsilon_{2}=\frac{\partial v}{\partial y}+w k_{2} \\
\epsilon_{6}=\frac{\partial u}{\partial y}+\frac{\partial v}{\partial x} & \kappa_{1}=\frac{\partial \psi_{x}}{\partial x} \\
\kappa_{2}=\frac{\partial \psi_{y}}{\partial y} & \kappa_{6}=\frac{\partial \psi_{x}}{\partial y}+\frac{\partial \psi_{y}}{\partial x} \\
\epsilon_{4}=\frac{\partial w}{\partial y}+\psi_{y} & \epsilon_{5}=\frac{\partial w}{\partial x}+\psi_{x}
\end{array}
$$

†On leave from the Department of Civil Engineering, Hefei University of Technology, 230009, Hefei, Anhui, P.R. China. 
in which $u, v$ and $w$ indicate the displacements of the midplane of the shells, $\psi_{x}$ and $\psi_{y}$ represent the rotations of the shells about $x$ and $y$ coordinate axes respectively. $k_{1}$ and $k_{2}$ are principal curvatures of shallow shells in $x_{1}$ and $x_{2}$ directions respectively.

The relations between the stress resultants and the strains are

$$
\begin{gathered}
N_{i}=A_{i j} \epsilon_{j}+B_{i j} \kappa_{j} \quad M_{i}=B_{i j} \epsilon_{j}+D_{i j} \kappa_{j} \\
\left(A_{i j}, B_{i j}, D_{i j}\right)=\sum_{k=1}^{N} \int_{h_{k-1}}^{h_{k}} Q_{i j}^{(k)}\left(1, z, z^{2}\right) \mathrm{d} z \quad(i, j=1,2,6) \\
Q_{1}=C_{45} \epsilon_{4}+C_{55} \epsilon_{5} \quad Q_{2}=C_{44} \epsilon_{4}+C_{45} \epsilon_{5} \\
C_{i j}=\sum_{k=1}^{N} \int_{h_{k-1}}^{h_{k}} Q_{i j}^{(k)} K_{i} K_{j} \mathrm{~d} z \quad(i, j=4,5)
\end{gathered}
$$

in which $h_{k}$ is the vertical distance from the midplane, $z=0$, to the upper surface of the $k$ th lamina. $K_{4}$ and $K_{5}$ are the shear correction factors [7,8]. $Q_{i j}^{(k)}$ are plane stress reduced stiffness coefficients of the $k$ th lamina $[7,8]$.

The equilibrium equations of the shells are

$$
\begin{array}{r}
\frac{\partial N_{1}}{\partial x}+\frac{\partial N_{6}}{\partial y}+q_{x}=0 \\
\frac{\partial N_{6}}{\partial x}+\frac{\partial N_{2}}{\partial y}+q_{y}=0 \\
\frac{\partial Q_{1}}{\partial x}+\frac{\partial Q_{2}}{\partial y}-N_{1} k_{1}-N_{2} k_{2}+q_{z}=0 \\
\frac{\partial M_{1}}{\partial x}+\frac{\partial M_{6}}{\partial y}-Q_{1}+m_{x}=0 \\
\frac{\partial M_{6}}{\partial x}+\frac{\partial M_{2}}{\partial y}-Q_{2}+m_{y}=0 .
\end{array}
$$

Substituting equations (1), (2) and (3) into (4), we obtain the following differential equations using the generalized displacements as basic unknowns.

$$
\Delta_{i j}^{*} U_{j}+P_{i}=0 \quad(i, j=1,2,3,4,5)
$$

where $U_{j}$ represents the displacements of the shallow shells in the direction of $x_{1}, x_{2}$ and $x_{3}$, and the rotations in the direction of $x_{1}$ and $x_{2}$, i.e. $U_{j}$ indicates $u, v, w, \psi_{x}$ and $\psi_{y} . P_{i}$ represents the generalized loads, i.e. $P_{i}$ indicates $q_{x}, q_{y}, q_{z}, m_{x}$ and $m_{y}$ respectively. $\Delta_{i j}^{*}$ is the differential operators which can be found in Ref. [8].

\section{FUNDAMENTAL SOLUTIONS}

According to the definition of fundamental solutions, the fundamental solutions of moderately thick laminated anisotropic shallow shells are a set of special solutions of equations (5) under the action of a set of unit point load, i.e.

$$
\Delta_{i j}^{*} U_{k j}^{*}(\zeta, \mathbf{x})=-\delta(\zeta, \mathbf{x}) \delta_{k i}
$$

in which $\delta(\zeta, \mathbf{x})$ is the Dirac $\delta$ function, $\zeta$ and $\mathbf{x}$ represent the coordinates of the source point and a field point respectively, $U_{k j}^{*}(\zeta, \mathbf{x})$ represents the generalized displacements in the $j$ direction at the field point $\mathbf{x}$ of an infinite shell when a unit point load is applied at the $k$ 
direction of the source point $\zeta$. Equation (6) is a set of partial differential equations. It is difficult to obtain its solutions directly. In this paper, we first use the method of plane wave decomposition to transfer equation (6) into a set of ordinary differential equations. We expand $U_{k j}^{*}(\zeta, \mathbf{x})$ and $\delta(\zeta, \mathbf{x})$ into a plane wave.

$$
\delta(\zeta, \mathbf{x})=-\frac{1}{4 \pi^{2}} \int_{0}^{2 \pi}|\rho|^{-2} \mathrm{~d} \theta, \quad U_{k j}^{*}(\zeta, \mathbf{x})=\int_{0}^{2 \pi} \tilde{U}_{k j}^{*}(\rho) \mathrm{d} \theta
$$

in which $\rho=\omega_{1}\left(x_{1}-\xi\right)+\omega_{2}\left(x_{2}-\eta\right),\left(\omega_{1}, \omega_{2}\right)$ are coordinates of a point on the unit circle, i.e. $\omega_{1}=\cos \theta, \omega_{2}=\sin \theta,\left(x_{1}, x_{2}\right)$ and $(\xi, \eta)$ are coordinates of a field point and the source point respectively. $\phi(\rho)$ is a function depending only on $\rho$. Substituting equation (7) into equation (6), and considering differential relationship $\frac{\partial}{\partial x_{\alpha}}=\omega_{\alpha} \frac{d}{d \rho}$, we have

$$
\tilde{\Delta}_{i j}^{*} \tilde{U}_{k j}^{*}(\rho)=\frac{1}{4 \pi^{2}}|\rho|^{-2} \delta_{k i}
$$

in which

$$
\begin{aligned}
& \tilde{\Delta}_{i j}^{*}=\left[\begin{array}{ccccc}
a_{11} \frac{\mathrm{d}^{2}}{\mathrm{~d} \rho^{2}} & a_{12} \frac{\mathrm{d}^{2}}{\mathrm{~d} \rho^{2}} & a_{13} \frac{\mathrm{d}}{\mathrm{d} \rho} & a_{14} \frac{\mathrm{d}^{2}}{\mathrm{~d} \rho^{2}} & a_{15} \frac{\mathrm{d}^{2}}{\mathrm{~d} \rho^{2}} \\
a_{12} \frac{\mathrm{d}^{2}}{\mathrm{~d} \rho^{2}} & a_{22} \frac{\mathrm{d}^{2}}{\mathrm{~d} \rho^{2}} & a_{23} \frac{\mathrm{d}}{\mathrm{d} \rho} & a_{24} \frac{\mathrm{d}^{2}}{\mathrm{~d} \rho^{2}} & a_{25} \frac{\mathrm{d}^{2}}{\mathrm{~d} \rho^{2}} \\
-a_{13} \frac{\mathrm{d}}{\mathrm{d} \rho} & -a_{23} \frac{\mathrm{d}}{\mathrm{d} \rho} & a_{33} \frac{\mathrm{d}^{2}}{\mathrm{~d} \rho^{2}}-a_{k} & a_{34} \frac{\mathrm{d}}{\mathrm{d} \rho} & a_{35} \frac{\mathrm{d}}{\mathrm{d} \rho} \\
a_{14} \frac{\mathrm{d}^{2}}{\mathrm{~d} \rho^{2}} & a_{24} \frac{\mathrm{d}^{2}}{\mathrm{~d} \rho^{2}} & -a_{34} \frac{\mathrm{d}}{\mathrm{d} \rho} & a_{44} \frac{\mathrm{d}^{2}}{\mathrm{~d} \rho^{2}}-C_{55} & a_{45} \frac{\mathrm{d}^{2}}{\mathrm{~d} \rho^{2}}-C_{45} \\
a_{15} \frac{\mathrm{d}^{2}}{\mathrm{~d} \rho^{2}} & a_{25} \frac{\mathrm{d}^{2}}{\mathrm{~d} \rho^{2}} & -a_{35} \frac{\mathrm{d}}{\mathrm{d} \rho} & a_{45} \frac{\mathrm{d}^{2}}{\mathrm{~d} \rho^{2}}-C_{45} & a_{55} \frac{\mathrm{d}^{2}}{\mathrm{~d} \rho^{2}}-C_{44}
\end{array}\right] \\
& a_{11}=A_{11} \omega_{1}^{2}+2 A_{16} \omega_{1} \omega_{2}+A_{66} \omega_{2}^{2} \quad a_{22}=A_{66} \omega_{1}^{2}+2 A_{26} \omega_{1} \omega_{2}+A_{22} \omega_{2}^{2} \\
& a_{12}=A_{16} \omega_{1}^{2}+\left(A_{12}+A_{66}\right) \omega_{1} \omega_{2}+A_{26} \omega_{2}^{2} \quad a_{14}=B_{11} \omega_{1}^{2}+2 B_{16} \omega_{1} \omega_{2}+B_{66} \omega_{2}^{2} \\
& a_{15}=B_{16} \omega_{1}^{2}+\left(B_{12}+B_{66}\right) \omega_{1} \omega_{2}+B_{26} \omega_{2}^{2} \quad a_{25}=B_{66} \omega_{1}^{2}+2 B_{26} \omega_{1} \omega_{2}+B_{22} \omega_{2}^{2} \\
& a_{33}=C_{55} \omega_{1}^{2}+2 C_{45} \omega_{1} \omega_{2}+C_{44} \omega_{2}^{2} \quad a_{44}=D_{11} \omega_{1}^{2}+2 D_{16} \omega_{1} \omega_{2}+D_{66} \omega_{2}^{2} \\
& a_{45}=D_{16} \omega_{1}^{2}+\left(D_{12}+D_{66}\right) \omega_{1} \omega_{2}+D_{26} \omega_{2}^{2} \quad a_{55}=D_{66} \omega_{1}^{2}+2 D_{26} \omega_{1} \omega_{2}+D_{22} \omega_{2}^{2} \\
& a_{k}=A_{11} k_{1}^{2}+2 A_{12} k_{1} k_{2}+A_{22} k_{2}^{2} \quad a_{24}=a_{15} \\
& a_{13}=\left(A_{11} k_{1}+A_{12} k_{2}\right) \omega_{1}+\left(A_{16} k_{1}+A_{26} k_{2}\right) \omega_{2} \\
& a_{23}=\left(A_{16} k_{1}+A_{26} k_{2}\right) \omega_{1}+\left(A_{12} k_{1}+A_{22} k_{2}\right) \omega_{2} \\
& a_{34}=\left(C_{55}-k_{1} B_{11}-k_{2} B_{12}\right) \omega_{1}+\left(C_{45}-k_{1} B_{16}-k_{2} B_{26}\right) \omega_{2} \\
& a_{35}=\left(C_{45}-k_{1} B_{16}-k_{2} B_{26}\right) \omega_{1}+\left(C_{44}-k_{1} B_{12}-k_{2} B_{22}\right) \omega_{2} \text {. }
\end{aligned}
$$

By the use of the Hörmander operator method, the solutions of equation (8) can be represented in the following from:

$$
\tilde{U}_{k j}^{*}(\rho)={ }^{\mathrm{co}} \tilde{\Delta}_{j k}^{*} \phi(\rho)
$$

where $\phi(\rho)$ is an unknown scalar function depending only on $\rho$, and ${ }^{\mathrm{c}} \tilde{\Delta}^{*}$ is the cofactor matrix of $\tilde{\Delta}^{*}$. Thus the fundamental solutions of the generalized displacements for moderately thick laminated anisotropic shallow shells are as follows. 


$$
\begin{aligned}
\tilde{U}_{\alpha \beta}^{*}(\rho) & =b_{\alpha \beta} D^{8} \phi(\rho)+d_{\alpha \beta} D^{6} \phi(\rho)+e_{\alpha \beta} D^{4} \phi(\rho)+f_{\alpha \beta} D^{2} \phi(\rho) \\
\tilde{U}_{\alpha 3}^{*}(\rho) & =-\tilde{U}_{3 \alpha}^{*}(\rho)=b_{\alpha} D^{7} \phi(\rho)+d_{\alpha} D^{5} \phi(\rho)+e_{\alpha} D^{3} \phi(\rho) \\
\tilde{U}_{\alpha 3+\beta}^{*}(\rho) & =\tilde{U}_{3+\beta \alpha}^{*}(\rho)=g_{\alpha \beta} D^{8} \phi(\rho)+h_{\alpha \beta} D^{6} \phi(\rho)+s_{\alpha \beta} D^{4} \phi(\rho) \\
\tilde{U}_{33}^{*}(\rho) & =A D^{8} \phi(\rho)+B D^{6} \phi(\rho)+C D^{4} \phi(\rho) \\
\tilde{U}_{33+\alpha}^{*}(\rho) & =-\tilde{U}_{3+\alpha 3}^{*}(\rho)=f_{\alpha} D^{7} \phi(\rho)+c_{\alpha} D^{5} \phi(\rho) \\
\tilde{U}_{\alpha+3 \beta+3}^{*}(\rho) & =q_{\alpha \beta} D^{8} \phi(\rho)+r_{\alpha \beta} D^{6} \phi(\rho)+t_{\alpha \beta} D^{4} \phi(\rho)
\end{aligned}
$$

in which $D^{k} \phi(\rho)=\frac{\mathrm{d}^{k} \phi(\rho)}{\mathrm{d} \rho^{k}},(k=1,2, \ldots), \alpha, b=1,2$.

$$
\begin{aligned}
& b_{11}=a_{33}\left(a_{22} \alpha_{1}+a_{24} \alpha_{2}+a_{25} \alpha_{3}\right) \\
& b_{22}=a_{33}\left(a_{11} \alpha_{1}+a_{14} \alpha_{18}+a_{15} \alpha_{19}\right) \\
& b_{12}=b_{21}=-a_{33}\left(a_{12} \alpha_{1}+a_{24} \alpha_{18}+a_{25} \alpha_{19}\right) \\
& d_{11}=a_{22}\left(a_{33} \alpha_{5}-a_{55} \alpha_{4}+a_{35} \alpha_{8}+a_{45} \alpha_{6}+a_{34} \alpha_{7}\right)+a_{23}\left(a_{23} \alpha_{1}+a_{24} \alpha_{7}+a_{25} \alpha_{8}\right) \\
& +a_{24}\left(a_{23} \alpha_{7}+a_{35} \alpha_{9}-a_{25} \alpha_{6}+a_{24} \alpha_{10}\right)+a_{25}\left(a_{23} \alpha_{8}+a_{34} \alpha_{11}+a_{25} \alpha_{4}-a_{24} \alpha_{6}\right) \\
& d_{22}=a_{11}\left(a_{33} \alpha_{5}-a_{55} \alpha_{4}+a_{35} \alpha_{8}+a_{45} \alpha_{6}+a_{34} \alpha_{7}\right)+a_{13}\left(a_{13} \alpha_{1}+a_{14} \alpha_{7}+a_{15} \alpha_{8}\right) \\
& +a_{14}\left(a_{13} \alpha_{7}+a_{35} \alpha_{20}-a_{15} \alpha_{6}+a_{14} \alpha_{10}\right)+a_{15}\left(a_{13} \alpha_{8}-a_{34} \alpha_{20}+a_{15} \alpha_{4}-a_{14} \alpha_{6}\right) \\
& d_{12}=d_{21}=-a_{12}\left(a_{33} \alpha_{5}-a_{55} \alpha_{4}+a_{35} \alpha_{8}+a_{45} \alpha_{6}+a_{34} \alpha_{7}\right)-a_{23}\left(a_{13} \alpha_{1}+a_{14} \alpha_{7}+a_{15} \alpha_{8}\right) \\
& -a_{24}\left(a_{13} \alpha_{7}+a_{35} \alpha_{20}-a_{15} \alpha_{6}+a_{14} \alpha_{10}\right)-a_{25}\left(a_{13} \alpha_{8}-a_{34} \alpha_{20}+a_{15} \alpha_{4}-a_{14} \alpha_{6}\right) \\
& e_{11}=a_{22}\left(C_{44} \alpha_{4}+a_{k} \alpha_{28}+a_{34} \alpha_{12}+a_{35} \alpha_{13}-C_{45} \alpha_{6}\right)+a_{23}\left(a_{24} \alpha_{12}+a_{25} \alpha_{13}-a_{23}\left(\alpha_{28}-\alpha_{5}\right)\right) \\
& +a_{24}\left(a_{23} \alpha_{12}+a_{k} \alpha_{14}\right)+a_{25}\left(a_{k} \alpha_{15}+a_{23} \alpha_{13}\right) \\
& e_{22}=a_{11}\left(C_{44} \alpha_{4}+a_{k} \alpha_{28}+a_{34} \alpha_{12}+a_{35} \alpha_{13}-C_{45} \alpha_{6}\right)+a_{13}\left(a_{14} \alpha_{12}+a_{15} \alpha_{13}-a_{13}\left(\alpha_{28}-\alpha_{5}\right)\right) \\
& +a_{14}\left(a_{13} \alpha_{12}+a_{k} \alpha_{21}\right)+a_{15}\left(a_{k} \alpha_{22}+a_{13} \alpha_{13}\right) \\
& e_{12}=e_{21}=-a_{12}\left(C_{44} \alpha_{4}+a_{k} \alpha_{28}+a_{34} \alpha_{12}+a_{35} \alpha_{13}-C_{45} \alpha_{6}\right)-a_{23}\left(a_{14} \alpha_{12}+a_{15} \alpha_{13}\right. \\
& \left.-a_{13}\left(\alpha_{28}-\alpha_{5}\right)\right)-a_{24}\left(a_{13} \alpha_{12}+a_{k} \alpha_{21}\right)-a_{25}\left(a_{k} \alpha_{22}+a_{13} \alpha_{13}\right) \\
& f_{11}=\alpha_{16} \alpha_{17} \quad f_{22}=\alpha_{23} \alpha_{17} \quad f_{12}=f_{21}=-\alpha_{24} \alpha_{17} \\
& b_{1}=-a_{12}\left(a_{23} \alpha_{1}+a_{24} \alpha_{7}+a_{25} \alpha_{8}\right)+a_{22}\left(a_{13} \alpha_{1}+a_{14} \alpha_{7}+a_{15} \alpha_{8}\right) \\
& +a_{24}\left(a_{13} \alpha_{2}+a_{35} \alpha_{25}-a_{23} \alpha_{18}\right)-a_{25}\left(-a_{13} \alpha_{3}+a_{34} \alpha_{25}+a_{23} \alpha_{19}\right) \\
& b_{2}=a_{11}\left(a_{23} \alpha_{1}+a_{24} \alpha_{7}+a_{25} \alpha_{8}\right)-a_{12}\left(a_{13} \alpha_{1}+a_{14} \alpha_{7}+a_{15} \alpha_{8}\right) \\
& -a_{14}\left(a_{13} \alpha_{2}+a_{35} \alpha_{25}-a_{23} \alpha_{18}\right)+a_{15}\left(-a_{13} \alpha_{3}+a_{34} \alpha_{25}+a_{23} \alpha_{19}\right) \\
& d_{1}=a_{12}\left(a_{23}\left(\alpha_{28}-\alpha_{5}\right)-a_{24} \alpha_{12}-a_{25} \alpha_{13}\right)-a_{22}\left(a_{13}\left(\alpha_{28}-\alpha_{5}\right)-a_{14} \alpha_{12}-a_{15} \alpha_{13}\right) \\
& +a_{24}\left(a_{23} \alpha_{21}-a_{13} \alpha_{14}\right)-a_{25}\left(a_{13} \alpha_{15}-a_{23} \alpha_{22}\right) \\
& d_{2}=-a_{11}\left(a_{23}\left(\alpha_{28}-\alpha_{5}\right)-a_{24} \alpha_{12}-a_{25} \alpha_{13}\right)+a_{12}\left(a_{13}\left(\alpha_{28}-\alpha_{5}\right)-a_{14} \alpha_{12}-a_{15} \alpha_{13}\right) \\
& -a_{14}\left(a_{23} \alpha_{21}-a_{13} \alpha_{14}\right)+a_{15}\left(a_{13} \alpha_{15}-a_{23} \alpha_{22}\right) \\
& e_{1}=\alpha_{26} \alpha_{17} \quad e_{2}=\alpha_{27} \alpha_{17} \\
& g_{11}=a_{33}\left(-a_{12} \alpha_{2}+a_{22} \alpha_{18}+a_{25} \alpha_{25}\right)
\end{aligned}
$$




$$
\begin{aligned}
& g_{12}=a_{33}\left(-a_{12} \alpha_{3}+a_{22} \alpha_{19}-a_{24} \alpha_{25}\right) \\
& g_{21}=a_{33}\left(a_{11} \alpha_{2}-a_{12} \alpha_{18}-a_{15} \alpha_{25}\right) \\
& g_{22}=a_{33}\left(a_{11} \alpha_{3}-a_{12} \alpha_{19}+a_{14} \alpha_{25}\right) \\
& h_{11}=-a_{12}\left(a_{23} \alpha_{7}+a_{24} \alpha_{10}+a_{35} \alpha_{9}-a_{25} \alpha_{6}\right)+a_{22}\left(a_{13} \alpha_{7}+a_{14} \alpha_{10}+a_{35} \alpha_{20}-a_{15} \alpha_{6}\right) \\
& -a_{23}\left(a_{13} \alpha_{2}-a_{23} \alpha_{18}+a_{35} \alpha_{25}\right)+a_{25}\left(a_{13} \alpha_{11}+a_{23} \alpha_{20}-a_{k} \alpha_{25}\right) \\
& h_{21}=a_{11}\left(a_{23} \alpha_{7}+a_{24} \alpha_{10}+a_{35} \alpha_{9}-a_{25} \alpha_{6}\right)-a_{12}\left(a_{13} \alpha_{7}+a_{14} \alpha_{10}+a_{35} \alpha_{20}-a_{15} \alpha_{6}\right) \\
& +a_{13}\left(a_{13} \alpha_{2}-a_{23} \alpha_{18}+a_{35} \alpha_{25}\right)-a_{15}\left(a_{13} \alpha_{11}+a_{23} \alpha_{20}-a_{k} \alpha_{25}\right) \\
& h_{12}=a_{12}\left(-a_{23} \alpha_{8}+a_{24} \alpha_{6}-a_{34} \alpha_{11}-a_{25} \alpha_{4}\right)-a_{22}\left(-a_{13} \alpha_{8}+a_{14} \alpha_{6}+a_{34} \alpha_{20}-a_{15} \alpha_{4}\right) \\
& +a_{23}\left(-a_{13} \alpha_{3}+a_{34} \alpha_{25}+a_{23} \alpha_{19}\right)-a_{24}\left(a_{13} \alpha_{11}+a_{23} \alpha_{20}-a_{k} \alpha_{25}\right) \\
& h_{22}=-a_{11}\left(-a_{23} \alpha_{8}+a_{24} \alpha_{6}-a_{34} \alpha_{11}-a_{25} \alpha_{4}\right)+a_{12}\left(-a_{13} \alpha_{8}+a_{14} \alpha_{6}+a_{34} \alpha_{20}-a_{15} \alpha_{4}\right) \\
& -a_{13}\left(-a_{13} \alpha_{3}+a_{34} \alpha_{25}+a_{23} \alpha_{19}\right)+a_{14}\left(a_{13} \alpha_{11}+a_{23} \alpha_{20}-a_{k} \alpha_{25}\right) \\
& s_{11}=-a_{12}\left(a_{23} \alpha_{12}+a_{k} \alpha_{14}\right)+a_{22}\left(a_{13} \alpha_{12}+a_{k} \alpha_{21}\right)+a_{23}\left(a_{13} \alpha_{14}-a_{23} \alpha_{21}\right) \\
& s_{21}=a_{11}\left(a_{23} \alpha_{12}+a_{k} \alpha_{14}\right)-a_{12}\left(a_{13} \alpha_{12}+a_{k} \alpha_{21}\right)-a_{13}\left(a_{13} \alpha_{14}-a_{23} \alpha_{21}\right) \\
& s_{12}=-a_{12}\left(a_{23} \alpha_{13}+a_{k} \alpha_{15}\right)+a_{22}\left(a_{13} \alpha_{13}+a_{k} \alpha_{22}\right)-a_{23}\left(a_{23} \alpha_{22}-a_{13} \alpha_{15}\right) \\
& s_{22}=a_{11}\left(a_{23} \alpha_{13}+a_{k} \alpha_{15}\right)-a_{12}\left(a_{13} \alpha_{13}+a_{k} \alpha_{22}\right)+a_{13}\left(a_{23} \alpha_{22}-a_{13} \alpha_{15}\right) \\
& A=a_{11}\left(a_{22} \alpha_{1}+a_{24} \alpha_{2}+a_{25} \alpha_{3}\right)-a_{12}\left(a_{12} \alpha_{1}+a_{25} \alpha_{19}+a_{24} \alpha_{18}\right) \\
& +a_{14}\left(a_{22} \alpha_{18}+a_{25} \alpha_{25}-a_{12} \alpha_{2}\right)-a_{15}\left(a_{12} \alpha_{3}-a_{22} \alpha_{19}-a_{24} \alpha_{25}\right) \\
& B=-a_{11}\left(a_{22}\left(\alpha_{28}-\alpha_{5}\right)+a_{24} \alpha_{14}+a_{25} \alpha_{15}\right)+a_{12}\left(a_{12}\left(\alpha_{28}-\alpha_{5}\right)+a_{14} \alpha_{14}+a_{15} \alpha_{15}\right) \\
& +a_{14}\left(a_{12} \alpha_{14}-a_{22} \alpha_{21}\right)-a_{15}\left(a_{22} \alpha_{22}-a_{12} \alpha_{15}\right) \\
& C=\alpha_{29} \alpha_{17} \\
& f_{1}=a_{11}\left(a_{22} \alpha_{7}-a_{23} \alpha_{2}-a_{25} \alpha_{9}\right)-a_{12}\left(a_{12} \alpha_{7}-a_{23} \alpha_{18}-a_{25} \alpha_{20}\right) \\
& +a_{13}\left(a_{12} \alpha_{2}-a_{22} \alpha_{18}-a_{25} \alpha_{25}\right)-a_{15}\left(a_{22} \alpha_{20}-a_{12} \alpha_{9}-a_{23} \alpha_{25}\right) \\
& f_{2}=a_{11}\left(a_{22} \alpha_{8}-a_{23} \alpha_{3}+a_{24} \alpha_{9}\right)-a_{12}\left(a_{12} \alpha_{8}-a_{23} \alpha_{19}+a_{24} \alpha_{20}\right) \\
& +a_{13}\left(a_{12} \alpha_{3}-a_{22} \alpha_{19}+a_{24} \alpha_{25}\right)-a_{14}\left(a_{12} \alpha_{9}-a_{22} \alpha_{20}+a_{23} \alpha_{25}\right) \\
& c_{1}=a_{11}\left(a_{23} \alpha_{14}+a_{22} \alpha_{12}\right)-a_{12}\left(a_{12} \alpha_{12}+a_{23} \alpha_{21}\right)+a_{13}\left(a_{22} \alpha_{21}-a_{12} \alpha_{14}\right) \\
& c_{2}=a_{11}\left(a_{23} \alpha_{15}+a_{22} \alpha_{13}\right)-a_{12}\left(a_{12} \alpha_{13}+a_{23} \alpha_{22}\right)+a_{13}\left(a_{22} \alpha_{22}-a_{12} \alpha_{15}\right) \\
& q_{11}=a_{33}\left(a_{25} \alpha_{30}+a_{15} \alpha_{31}-a_{55} \alpha_{29}\right) \quad q_{22}=a_{33}\left(a_{25} \alpha_{32}+a_{14} \alpha_{33}-a_{44} \alpha_{29}\right)
\end{aligned}
$$




$$
\begin{aligned}
q_{12}= & a_{21}=a_{33}\left(a_{45} \alpha_{29}-a_{24} \alpha_{30}-a_{14} \alpha_{31}\right) \\
r_{11}= & a_{55}\left(a_{12} \alpha_{24}-a_{11} \alpha_{16}-a_{13} \alpha_{26}\right)-a_{35}\left(a_{25} \alpha_{27}+a_{35} \alpha_{29}+a_{15} \alpha_{26}\right) \\
& +a_{25}\left(a_{25} \alpha_{23}-a_{15} \alpha_{24}-a_{35} \alpha_{27}\right)-a_{15}\left(a_{35} \alpha_{26}+a_{25} \alpha_{24}-a_{15} \alpha_{16}\right)+a_{33} C_{44} \alpha_{29} \\
r_{22}= & a_{44}\left(a_{12} \alpha_{24}-a_{11} \alpha_{16}-a_{13} \alpha_{26}\right)-a_{34}\left(a_{24} \alpha_{27}+a_{14} \alpha_{26}+a_{34} \alpha_{29}\right) \\
& +a_{24}\left(a_{24} \alpha_{23}-a_{14} \alpha_{24}-a_{34} \alpha_{27}\right)-a_{14}\left(a_{34} \alpha_{26}+a_{24} \alpha_{24}-a_{14} \alpha_{16}\right)+a_{33} C_{55} \alpha_{29} \\
r_{12}= & r_{21}=-a_{45}\left(a_{12} \alpha_{24}-a_{11} \alpha_{16}-a_{13} \alpha_{26}\right)+a_{35}\left(a_{24} \alpha_{27}+a_{34} \alpha_{29}+a_{14} \alpha_{26}\right) \\
& -a_{25}\left(a_{24} \alpha_{23}-a_{14} \alpha_{24}-a_{34} \alpha_{27}\right)+a_{15}\left(a_{34} \alpha_{26}+a_{24} \alpha_{24}-a_{14} \alpha_{16}\right)-a_{33} C_{45} \alpha_{29} \\
& t_{11}=C_{44}\left(a_{11} \alpha_{16}-a_{12} \alpha_{24}+a_{13} \alpha_{26}\right)
\end{aligned}
$$

Substituting equation (11) into equation (8), we obtain a tenth order ordinary differential equation, which can be written in the following form.

$$
\frac{\mathrm{d}^{4}}{\mathrm{~d} \rho^{4}}\left(\frac{\mathrm{d}^{6}}{\mathrm{~d} \rho^{6}}+a_{1} \frac{\mathrm{d}^{4}}{\mathrm{~d} \rho^{4}}+a_{2} \frac{\mathrm{d}^{2}}{\mathrm{~d} \rho^{2}}+a_{3}\right) \phi(\rho)=\frac{1}{4 \pi^{2}|\rho|^{2} A_{1}}
$$

in which

$$
\begin{aligned}
& A_{1}=a_{11} b_{11}+a_{12} b_{12}+a_{14} g_{11}+a_{15} g_{12} \\
& A_{2}=a_{11} d_{11}+a_{12} d_{12}+a_{13} b_{1}+a_{14} h_{11}+a_{15} h_{12} \\
& A_{3}=a_{11} e_{11}+a_{12} e_{12}+a_{13} d_{1}+a_{14} s_{11}+a_{15} s_{12} \\
& A_{4}=a_{11} f_{11}+a_{12} f_{12}+a_{13} e_{1} \\
& a_{1}=\frac{A_{2}}{A_{1}} \quad a_{2}=\frac{A_{3}}{A_{1}} \quad a_{3}=\frac{A_{4}}{A_{1}} .
\end{aligned}
$$

Integrating equation (14) for four times, deleting the constants of integration $[3,9]$ and by use 
of the method of variation of arbitrary parameters, unknown function $\phi(\rho)$ can be obtained, which is of the form:

$$
\begin{aligned}
\phi(\rho)= & 2 \sum_{i=1}^{3} \lambda_{i}\left(p_{i}^{2} \rho^{2} \ln |\rho|+2 \ln |\rho|+3\right) \\
& +2 \sum_{i=1}^{3} \lambda_{i}\left(\mathrm{e}^{\rho_{i} \rho} \int_{\rho}^{\infty} \frac{\mathrm{e}^{-p_{i} \sigma}}{\sigma} \mathrm{d} \sigma-\mathrm{e}^{-p_{i} \rho} \int_{-\infty}^{\rho} \frac{\mathrm{e}^{p_{i} \sigma}}{\sigma} \mathrm{d} \sigma\right)
\end{aligned}
$$

where

$$
\begin{aligned}
\lambda_{1} & =\frac{1}{16 \pi^{2} A_{1}\left(p_{1}^{2}-p_{2}^{2}\right)\left(p_{1}^{2}-p_{3}^{2}\right) p_{1}^{4}}, \quad \lambda_{2}=\frac{1}{16 \pi^{2} A_{1}\left(p_{2}^{2}-p_{1}^{2}\right)\left(p_{2}^{2}-p_{3}^{2}\right) p_{2}^{4}} \\
\lambda_{3} & =\frac{1}{16 \pi^{2} A_{1}\left(p_{3}^{2}-p_{1}^{2}\right)\left(p_{3}^{2}-p_{2}^{2}\right) p_{3}^{4}}, \quad p_{1}=\left(\eta_{1}+\eta_{2}-\frac{1}{3} a_{1}\right)^{1 / 2} \\
p_{2} & =\frac{1}{\sqrt{2}}\left(\sqrt{r+\xi_{1}}+i \sqrt{r-\xi_{2}}\right), \quad p_{3}=\frac{1}{\sqrt{2}}\left(\sqrt{r+\xi_{1}}-i \sqrt{r-\xi_{2}}\right) \\
r & =\left(\xi_{1}^{2}+\xi_{2}^{2}\right)^{1 / 2}, \quad \xi_{1}=-\frac{1}{2}\left(\eta_{1}+\eta_{2}\right)-\frac{1}{3} a_{1} \\
\xi_{2} & =\frac{\sqrt{3}}{2}\left(\eta_{1}-\eta_{2}\right), \quad \eta_{1}=\left(-\frac{q}{2}+\sqrt{b}\right)^{1 / 3} \\
\eta_{2} & =\left(-\frac{q}{2}-\sqrt{b}\right)^{1 / 3}, \quad q=\left(\frac{2}{27} a_{1}^{3}-\frac{1}{3} a_{1} a_{2}+a_{3}\right) \\
b & =\frac{1}{4} a_{3}^{2}+\frac{1}{27}\left(a_{2}^{3}+a_{1}^{3} a_{3}\right)-\frac{1}{108} a_{1}^{2} a_{2}^{2}-\frac{1}{6} a_{1} a_{2} a_{3} .
\end{aligned}
$$

In order to obtain the fundamental solutions, it is necessary to calculate the function $\phi(\rho)$ and its derivatives. For simplicity, we introduce the following two functions.

$$
\begin{aligned}
& A_{i}\left(p_{i} \rho\right)=\mathrm{e}^{p i \rho} \int_{\rho}^{\infty} \frac{\mathrm{e}^{-p_{i} \sigma}}{\sigma} \mathrm{d} \sigma-\mathrm{e}^{-p_{i} \rho} \int_{-\infty}^{\rho} \frac{\mathrm{e}^{p_{i} \sigma}}{\sigma} \mathrm{d} \sigma \\
& B_{i}\left(p_{i} \rho\right)=\mathrm{e}^{p i \rho} \int_{\rho}^{\infty} \frac{\mathrm{e}^{-p_{i} \sigma}}{\sigma} \mathrm{d} \sigma+\mathrm{e}^{-p_{i} \rho} \int_{-\infty}^{\rho} \frac{\mathrm{e}^{p_{i} \sigma}}{\sigma} \mathrm{d} \sigma \quad(i=1,2,3) .
\end{aligned}
$$

The above two functions have the following differential relations:

$$
\frac{\mathrm{d} A_{i}\left(p_{i} \rho\right)}{\mathrm{d} \rho}=p_{i} B_{i}\left(p_{i} \rho\right)-\frac{2}{\rho}, \quad \frac{\mathrm{d} B_{i}\left(p_{i} \rho\right)}{\mathrm{d} \rho}=p_{i} A_{i}\left(p_{i} \rho\right) .
$$

Using the expression of exponential integral [10], we have

$$
\begin{gathered}
A_{1}\left(p_{1} \rho\right)=\mathrm{e}^{p_{1} \rho} E_{1}\left(p_{1} \rho\right)-\mathrm{e}^{-p_{1} \rho} E_{i}\left(p_{1} \rho\right) \\
B_{1}\left(p_{1} \rho\right)=\mathrm{e}^{p_{1} \rho} E_{1}\left(p_{1} \rho\right)+\mathrm{e}^{-p_{1} \rho} E_{i}\left(p_{1} \rho\right) \text { for } \rho>0 \\
A_{1}\left(p_{1} \rho\right)=-\mathrm{e}^{\left|\rho_{1} \rho\right|} E_{1}\left(\left|p_{1} \rho\right|\right)+\mathrm{e}^{\left|p_{1} \rho\right|} E_{1}\left(\left|p_{1} \rho\right|\right) \\
B_{1}\left(p_{1} \rho\right)=-\mathrm{e}^{\left|p_{1} \rho\right|} E_{1}\left(\left|p_{1} \rho\right|\right)-\mathrm{e}^{\left|p_{1} \rho\right|} E_{1}\left(\left|p_{1} \rho\right|\right) \quad \text { for } \rho<0 \\
A_{2}\left(p_{2} \rho\right)=\mathrm{e}^{p_{2} \rho}\left(E_{1}\left(p_{2} \rho\right)-\frac{\pi}{2} i(1-\operatorname{sgn} \rho)\right)-\mathrm{e}^{-p_{2} \rho}\left(-E_{1}\left(-p_{2} \rho\right)+\frac{\pi}{2} i(1+\operatorname{sgn} \rho)\right) \\
B_{2}\left(p_{2} \rho\right)=\mathrm{e}^{p_{2} \rho}\left(E_{1}\left(p_{2} \rho\right)-\frac{\pi}{2} i(1-\operatorname{sgn} \rho)\right)+\mathrm{e}^{-p_{2} \rho}\left(-E_{1}\left(-p_{2} \rho\right)+\frac{\pi}{2} i(1+\operatorname{sgn} \rho)\right) .
\end{gathered}
$$


Because of $\vec{p}_{2} \rho=p_{3}$, we have $A_{3}\left(p_{3} \rho\right)=A_{3}\left(\bar{p}_{2} \rho\right)$ and $B_{3}\left(p_{3} \rho\right)=B_{3}\left(\bar{p}_{2} \rho\right)$. According to the property of complex variable function, there are the following relations.

$$
A_{3}\left(p_{3} \rho\right)=\overline{A_{2}\left(p_{2} \rho\right)}, \quad B_{3}\left(p_{3} \rho\right)=\overline{B_{2}\left(p_{2} \rho\right)} .
$$

As long as $A_{2}\left(p_{2} \rho\right)$ and $B_{2}\left(p_{2} \rho\right)$ are obtained, we can calculate $A_{3}\left(p_{2} \rho\right)$ and $B_{3}\left(p_{2} \rho\right)$ by use of the above equations. In what follows, we only consider the calculation of $A_{1}\left(p_{2} \rho\right), B_{1}\left(p_{2} \rho\right)$, $A_{2}\left(p_{2} \rho\right)$ and $B_{2}\left(p_{2} \rho\right)$. Using the series expression of the exponential function and exponential integral [10], we have

$$
\begin{gathered}
A_{1}\left(p_{1} \rho\right)=-2 \sum_{n=0}^{\infty} \frac{\left(p_{1} \rho\right)^{2 n}}{(2 n) !}\left(\gamma+\ln \left|p_{1} \rho\right|-F(2 n+1)\right) \\
B_{1}\left(p_{1} \rho\right)=-2 \sum_{n=1}^{\infty} \frac{\left(p_{1} \rho\right)^{2 n-1}}{(2 n-1) !}\left(\gamma+\ln \left|p_{1} \rho\right|-F(2 n)\right) \\
A_{2}\left(p_{2} \rho\right)=-2 \sum_{n=0}^{\infty} \frac{\left(p_{2} \rho\right)^{2 n}}{(2 n) !}\left(\gamma+\ln \left|p_{2} \rho\right|+i\left(\frac{\pi}{2}-\alpha\right)-F(2 n+1)\right) \\
B_{2}\left(p_{2} \rho\right)=-2 \sum_{n=1}^{\infty} \frac{\left(p_{2} \rho\right)^{2 n-1}}{(2 n-1) !}\left(\gamma+\ln \left|p_{2} \rho\right|+i\left(\frac{\pi}{2}-\alpha\right)-F(2 n)\right)
\end{gathered}
$$

where $\gamma(=0.57721 \cdots)$ is the Euler constant.

$$
\alpha=\arctan \left|\frac{\sqrt{r+\xi_{1}}}{\sqrt{r-\xi_{1}}}\right|, \quad F(m+1)=\sum_{s=1}^{m} \frac{1}{s}, \quad F(1)=0 .
$$

Using equations (11) and (12), we obtain $\phi(\rho)$ and its derivatives as follows:

$$
\begin{aligned}
\phi(\rho) & =2 \sum_{i=1}^{3} \lambda_{i}\left(p_{i}^{2} \rho^{2} \ln |\rho|+(2 \ln |\rho|+3)+A_{i}\left(p_{i} \rho\right)\right) \\
D \phi(\rho) & =2 \sum_{i=1}^{3} \lambda_{i}\left(2 p_{i}^{2} \rho \ln |\rho|+p_{i}^{2} \rho+p_{i} B_{i}\left(p_{i} \rho\right)\right) \\
D^{2} \phi(\rho) & =2 \sum_{i=1}^{3} \lambda_{i}\left(2 p_{i}^{2} \ln |\rho|+3 p_{i}^{2}+p_{i}^{2} A_{i}\left(p_{i} \rho\right)\right) \\
D^{3} \phi(\rho) & =2 \sum_{i=1}^{3} \lambda_{i} p_{i}^{3} B_{i}\left(p_{i} \rho\right), \quad D^{4} \phi(\rho)=2 \sum_{i=1}^{3} \lambda_{i} p_{i}^{4} A_{i}\left(p_{i} \rho\right) \\
D^{5} \phi(\rho) & =2 \sum_{i=1}^{3} \lambda_{i} p_{i}^{5} B_{i}\left(p_{i} \rho\right), \quad D^{6} \phi(\rho)=2 \sum_{i=1}^{3} \lambda_{i} p_{i}^{6} A_{i}\left(p_{i} \rho\right) \\
D^{7} \phi(\rho) & =2 \sum_{i=1}^{3} \lambda_{i} p_{i}^{7} B_{i}\left(p_{i} \rho\right), \\
D^{9} \phi(\rho) & =2 \sum_{i=1}^{3} \lambda_{i} p_{i}^{9} B_{i}\left(p_{i} \rho\right)-\frac{1}{4 \pi^{2} A_{1} \rho} \\
D^{10} \phi(\rho) & =2 \sum_{i=1}^{3} \lambda_{i} p_{i}^{10} A_{i}\left(p_{i} \rho\right)+\frac{1}{4 \pi^{2} A_{1} \rho^{2}} .
\end{aligned}
$$

In the procedure of obtaining equations (25), we have used the following relations:

$$
\sum_{i=1}^{3} \lambda_{i} p_{i}^{4}=0, \quad \sum_{i=1}^{3} \lambda_{i} p_{i}^{6}=0, \quad \sum_{i=1}^{3} \lambda_{i} p_{i}^{8}=\frac{1}{16 \pi^{2} A_{1}} .
$$

The computation of $\phi(\rho)$ and its derivatives is reduced to the calculation of $A_{1}\left(p_{i} \rho\right), B_{1}\left(p_{i} \rho\right)$, $A_{2}\left(p_{i} \rho\right)$ and $B_{2}\left(p_{i} \rho\right)$. Taking a few terms of equations (22) and (23), we can obtain quite 
results for $A_{1}\left(p_{i} \rho\right)$, etc. when $\left|p_{i} \rho\right|$ is small. Using the asymptotic representation of exponential integral $E_{1}$ and $E_{i}$ in Refs [10-12] and combining equations (19) and (20), we can calculate $A_{\mathrm{t}}\left(p_{i} \rho\right)$, etc. when $\left|p_{i} \rho\right|$ is large. Using the above described method, we can obtain a good result for the calculation of $A_{1}\left(p_{i} \rho\right), B_{1}\left(p_{i} \rho\right), A_{2}\left(p_{i} \rho\right)$ and $B_{2}\left(p_{i} \rho\right)$. In the calculation of the fundamental solutions, we have to deal with the following integral:

$$
I_{1}=\int_{0}^{2 \pi} F_{1}(\theta) \frac{\mathrm{d}^{k} \phi(\rho)}{\mathrm{d} \rho^{k}}, \quad(k=1,2,3, \ldots, 9) .
$$

In the range between 0 and $2 \pi$, the integrand has two points which make $\rho=0$. We first determine values $\theta_{0}$ which make $\rho=0$ and then split $(0,2 \pi]$ into four intervals. As the integrand is a periodic function, the four intervals can be written in the following form:

$$
\begin{gathered}
\left(\theta_{0}, \theta_{0}+\frac{\pi}{2}\right),\left(\theta_{0}+\frac{\pi}{2}, \theta_{0}+\pi\right) \\
\left(\theta_{0}+\pi, \theta_{0}+\frac{3 \pi}{2}\right),\left(\theta_{0}+\frac{3 \pi}{2}, \theta_{0}+2 \pi\right) .
\end{gathered}
$$

We can calculate the integral $I_{1}$ on each interval with any numerical integral method. The value $\theta_{0}$ can be determined by the following equation:

$$
\theta_{0}=\arctan \left(-\frac{x-\xi}{y-\eta}\right)
$$

Up to now, we have obtained the computational formulation of the fundamental solutions of the generalized displacements. Substituting equations (7) and (12) into equation (2) and using equations (1) and (3), we can obtain the fundamental solutions of the generalized forces for moderately thick laminated anisotropic shallow shells.

\section{CONCLUSIONS}

The fundamental solutions of moderately thick laminated anisotropic shallow shells have been presented in a definite integral form. They can be used to analyze the distribution of stresses and displacements in the neighborhood of the singular point which a concentrated force is applied at. They can also be taken as the kernel function of boundary integral equation method and used to analyze the static and dynamic problems of the shells. The boundary element analysis of moderately thick laminated anisotropic shallow shells will be given in successive paper.

Acknowledgements-The first author is grateful for the financial support of the Alexander von Humboldt Foundation in Germany and the National Natural Science Foundation of P.R. China.

\section{REFERENCES}

[1] S. LUKASIEWICZ, Local Loads in Plates and Shells. Noordhoff I. P., Loydon (1979).

[2] J. G. WANG and M. HUANG, Acta Mech. Sinica 7, 258 (1991).

[3] J. G. WANG, Acta Mech. 94, 113 (1992).

[4] L. HÖRMANDER, Linear Partial Differential Operators. Springer, Berlin (1976).

[5] I. M. GEL'FAND and G. E. SHILOV, Generalized Functions, Vol. 1. Academic Press, New York (1967).

[6] J. G. WANG and M. HUANG, Acta Mekriae Compositae Sinica 9, 71 (1992) (in Chinese).

[7] J. R. VINSON and T. W. CHOU, Composite Materials and Their Use in Structures. Applied Science, London (1975).

[8] Z. WANG, Mechanics of Composite Materials and Structural Mechanics of Composite Materials. Mechanical Industrial Press (1991) (in Chinese).

[9] T. MATSUI and O. MATSUOKS, Int. J. Solids and Structures 14, 971 (1976). 
[10] M. ABRAMOWITZ and I. A. STEGUN, Handbook of Mathematical Functions. U.S. Government Printing Office (1965)

[11] W. J. CODY and H. C. THACHER, Math. Comput. 22, 641 (1968).

[12] W. J. CODY and H. C. THACHER, Math. Comput. 23, 289 (1969).

(Received 18 July 1994; accepted 3 October 1994) 